\title{
Estimating Activity Coefficients of Target Components in Poorly Specified Mixtures with NMR Spectroscopy and COSMO-RS
}

\author{
Thomas Specht, Kerstin Münnemann, Fabian Jirasek*, Hans Hasse \\ Laboratory of Engineering Thermodynamics (LTD), TU Kaiserslautern, \\ Erwin-Schrödinger-Straße 44, 67663 Kaiserslautern, Germany
}

\begin{abstract}
Poorly specified mixtures are common in process engineering, especially in bioprocess engineering. The properties of such mixtures of unknown composition cannot be described using conventional thermodynamic models. The NEAT method, which has recently been developed in our group, enables the calculation of activity coefficients of known target components in such poorly specified mixtures. In NEAT, the group composition of the mixture is determined by NMR spectroscopy and a thermodynamic group contribution method is used for calculating the activity coefficients. In all previous studies with NEAT, the UNIFAC group contribution method was used. In the present work, we demonstrate that NEAT can also be applied with another important method for predicting activity coefficients: COSMO-RS. COSMO-RS (OL) developed in Oldenburg together with its group contribution version GC-COSMO-RS (OL) is used here. The new version of NEAT was successfully tested. For a variety of aqueous mixtures excellent agreement of the NEAT predictions, for which only information on the target component was used, with results that were obtained using the full knowledge on the composition of the mixture was found. The results demonstrate the generic nature of the idea of NEAT and the broad applicability of the method.
\end{abstract}

\footnotetext{
${ }^{*}$ Corresponding author

Email address: fabian.jirasek@mv.uni-kl.de (Fabian Jirasek)
}

Preprint submitted to Fluid Phase Equilibria

February 13, 2020 
Keywords: NEAT, COSMO-RS, NMR spectroscopy, Activity coefficients, Unknown composition

\section{Introduction}

The separation of target components from complex liquid mixtures is an ubiquitous task in process engineering. Fluid separation processes, such as distillation, extraction, and crystallization, are based on concentration differences 5 in coexisting phases and are often modeled using the equilibrium stage concept. Hereby information on the activity coefficients of the target components in the liquid phase is needed for the calculation of the phase equilibrium. Since the experimental determination of activity coefficients is tedious, prediction methods have been developed, of which the most widely used are UNIFAC [1 3$]$ and COSMO-RS 4, 5. UNIFAC is a group contribution version of the $G^{\mathrm{E}}$ model UNIQUAC [6]. COSMO-RS is basically also a $G^{\mathrm{E}}$ model, in which the energetic part is described based on quantum-chemical calculations. The key element in this is the determination of the $\sigma$-profile that represents the surface charges on a cavity in a dielectric medium, in which the target molecule is embedded [7.

15 All established thermodynamic models, including UNIFAC and COSMO-RS, have in common, that they can only be applied to mixtures of which the full speciation is known. However, there are many situations in which the full speciation of a mixture is not available. In bioprocess engineering, for example, this is the rule rather than an exception. Consider fermentation broths as an example: they often contain a huge number of unknown components and it is practically infeasible to fully elucidate their specification.

In the following, mixtures that contain components of unknown type and/or unknown concentration are called poorly specified mixtures. It is however assumed that each poorly specified mixture contains at least one component of which the nature and the concentration is known. We will call these target components and present a method, with which their activity coefficient in the poorly specified mixture can be calculated without having to know anything 
about unknown components of the mixture.

For elucidation and quantification of the unknown components in a poorly specified mixture, analytical methods such as NMR spectroscopy can be used. However, for complex mixtures, the application of these methods for a full elucidation is often much too time consuming and in many cases even impossible, e.g. due to overlapping or unclear assignment of peaks.

To circumvent these problems, many authors introduce pseudo components in their models of poorly specified mixtures. This approach has, e.g., often been used for modeling petrochemical systems [8 16. The drawback of this approach is that ad hoc assumptions on the pseudo components are needed, which, however, influence the results. This makes predictions cumbersome.

Therefore, the NEAT method (NMR Spectroscopy for the Estimation of Activity 40 Coefficients of Target Components in Poorly Specified Mixtures) was developed in previous works of our group [17, 18. NEAT is based on a combination of NMR spectroscopy and a thermodynamic group contribution method and enables the prediction of the activity coefficient $\gamma_{\mathrm{T}}$ of a target component $\mathrm{T}$ in a poorly specified mixture based only on information on the target component $\mathrm{T}$ 45 and a single NMR spectrum of the mixture. In NEAT, information on the type and concentration of the chemical groups of the unknown components in the poorly specified mixture are obtained from the NMR spectrum of the mixture. This is much easier than obtaining the respective information on the components. The information on the groups is then used in a thermodynamic group contribution method for calculating the activity coefficient of the target component $\mathrm{T}$. It has been shown that the results obtained with NEAT for the poorly specified mixture often match those obtained for the fully specified mixture remarkably well [17, 18. This has been demonstrated for aqueous, non-aqueous, and also for reactive mixtures. Furthermore, it has been shown that, using only 55 a single NMR spectrum, NEAT can be successfully applied for predicting activity coefficients for any mixtures, as long as the composition of the unknown components remains constant [19]. This is, for instance, practically relevant for describing the selective removal of the target component $\mathrm{T}$ or the selective re- 
moval of a known solvent from the mixture. For a complete description of phase equilibria, reliable information on the activity coefficients of all components in the considered mixture are needed. However, the activity coefficient $\gamma_{\mathrm{T}}$ of the target component is directly related to the affinity of the target component to a second phase. In a recent study, we have demonstrated how NEAT can thus be applied for the conceptual design of liquid-liquid extraction processes 20]. group contribution method was used: UNIFAC. In this work, we demonstrate that NEAT is a generic framework that is not restricted to the use of UNIFAC, but can in principle be combined with any group contribution method. In all cases, the quality of the NEAT results is inherently limited by the quality of the underlying thermodynamic group contribution method. It would only be by chance that the predictions of experimental data obtained from NEAT would be better than those obtained from the group contribution method (assuming the composition of the mixture would be known). It is therefore interesting to study the application of different thermodynamic group contributions methods 75 in NEAT.

We have therefore extended our previous studies and investigated how COSMORS, which is one of the most important models for predicting activity coefficients besides UNIFAC, can be used within the NEAT framework. In its original version, COSMO-RS [4, 5, 7] is not a group contribution method. However, group contribution methods have been developed, that enable an estimation of $\sigma$-profiles, cavity surface areas $A$, and cavity volumes $V$ without having to perform the quantum-chemical calculation. Such a group contribution version of COSMO-RS is used in the present work. Furthermore, there are different versions of the COSMO-RS $G^{\mathrm{E}}$ model, some of which have not been fully disclosed. In the present work, we use the group contribution version of COSMORS (OL) 21] as it was presented by the group of Gmehling in Oldenburg [22]. It is designated in the following as GC-COSMO-RS (OL). It is combined here with results from ${ }^{13} \mathrm{C}$ NMR spectroscopy from which the group composition of the studied mixtures was obtained in the same way as in our previous work [18]. 
90 The new method is tested using several poorly specified mixtures of the type known target component $\mathrm{T}+$ unknown components $\mathrm{U}+$ solvent water $\mathrm{W}$ as test cases. In all cases, information on the unknown components in the mixtures were not used for the predictions with NEAT but only for comparison of the predictions with results for the fully specified mixtures. New NMR measurements were carried out only for some of these mixtures. The other cases were already studied in our previous work [17] and the NMR spectroscopic results were simply re-evaluated to obtain information on the group speciation that could be used together with GC-COSMO-RS (OL). For all systems, the predictions obtained with the present version of NEAT (NMR + GC-COSMO$\mathrm{RS}(\mathrm{OL})$ ) were compared to results that were obtained using the full knowledge of the speciation of the mixture. Additionally, in one case the predictions of the newly introduced version of NEAT are compared to predictions of the recently described version of NEAT (NMR + modified UNIFAC (Dortmund) [2, 17 20] as well as with results that were obtained with the respective $G^{\mathrm{E}}$ models using the full knowledge of the speciation of the mixture.

\section{Materials and Methods}

\subsection{Materials}

For all mixtures prepared in this work, ultra pure water, which was produced with a water purification system of Merck Millipore (Elix Essential 5), was used.

Information on all other chemicals that were used in the experiments in this work is given in Table 1 . 
Table 1: Suppliers and purities of chemicals used in this work. Purities according to supplier's specification.

\begin{tabular}{l|l|l|l}
\hline \hline Chemical & Formula & Supplier & Purity \\
\hline Acetic acid & $\mathrm{C}_{2} \mathrm{H}_{4} \mathrm{O}_{2}$ & Carl Roth & $\geq 99.80 \%$ \\
Acetone & $\mathrm{C}_{3} \mathrm{H}_{6} \mathrm{O}$ & Fisher Chemical & $\geq 99.97 \%$ \\
Cyclohexanone & $\mathrm{C}_{6} \mathrm{H}_{10} \mathrm{O}$ & Sigma Aldrich & $\geq 99.80 \%$ \\
Ethanol & $\mathrm{C}_{2} \mathrm{H}_{6} \mathrm{O}$ & Merck & $\geq 99.90 \%$ \\
2-Propanol & $\mathrm{C}_{3} \mathrm{H}_{8} \mathrm{O}$ & Sigma Aldrich & $\geq 99.90 \%$ \\
TMSP-d4 & $\mathrm{NaC}_{6} \mathrm{H}_{9} \mathrm{D}_{4} \mathrm{O}_{2} \mathrm{Si}$ & Deutero & $\geq 98.00 \%$ \\
\hline \hline
\end{tabular}

\subsection{Methods}

\subsubsection{Sample preparation and NMR spectroscopy}

All sample mixtures were prepared gravimetrically in $20 \mathrm{ml}$ glass vessels. The mass of each sample was approximately $10 \mathrm{~g}$. To each sample, a small amount of sodium 3-(trimethylsilyl)tetradeuteriopropionate (TMSP-d4) was added as NMR standard. After thoroughly shaking the samples to obtain homogenous solutions, $1 \mathrm{ml}$ of each sample was transferred to a $5 \mathrm{~mm}$ NMR vial. Quantitative inverse-gated ${ }^{13} \mathrm{C}$ NMR spectra were recorded with a $400 \mathrm{MHz} \mathrm{NMR}$ spectrometer from Bruker (Avance) with a cryogenic probe (pulse angle: 60 acquisition time: 1.5 - $5.6 \mathrm{~s}$, number of scans: 64 or 128, Relaxation Delay: 45 $65 \mathrm{~s}$ ). Software from MestReLabs (MNova) was used for baseline correction, phase correction, and quantitative evaluation. Direct integration turned out to be sufficient in all cases, as basically only spectral areas, which were assigned to groups, had to be evaluated. The chemical shift refers to the TMSP-d4 peak.

\subsubsection{Assignment and quantification of chemical groups}

The composition of all studied mixtures was known from sample preparation. However, this information was not used for the predictions with NEAT, but only for testing the quality of predictions. For the predictions with NEAT, 

get component $\mathrm{T}+$ unknown components $\mathrm{U}+$ water $\mathrm{W}$. Only the nature and the mass fraction of the target component were assumed to be known. Information on the unknown components $U$ was obtained from the NMR spectrum of the mixture. As comprehensively described in our previous work [18], this includes the assignment of chemical groups to regions of chemical shift in the NMR spectrum. The group assignment used for the evaluation of the NMR spectra will in general not be completely identical with the group list of the thermodynamic group contribution method. Hence, a mapping is needed. As the group lists of modified UNIFAC (Dortmund), in the following abbreviated as UNIFAC (DO), and GC-COSMO-RS (OL) are not identical, the group assignment cannot simply be adopted from our previous work [18] but has to be reconsidered. The assignment used in this work for the predictions with NEAT based on GC-COSMO-RS (OL) is given in Table2, The group assignment used in this work for the predictions with NEAT based on UNIFAC (DO), which is shown in Table 3 , is similar, but not identical with the one used our previous work [18. The only difference is that 'CH3' and 'CH2' groups were distinguished here as it was observed that these groups can easily be distinguished in most spectra that were evaluated. The mass fraction of the groups in the mixture was obtained from the integration over the chemical shift regions. All identified groups were lumped to a mean unknown component $\tilde{U}$ to which a molar mass of $150 \mathrm{~g} \mathrm{~mol}^{-1}$ was assigned, as suggested in our previous works [17, 18]. The mass fraction of water $\mathrm{W}$ in the poorly specified mixture was calculated from the mass balance. In another version of NEAT, for which results are provided in the Supporting Information, information on the mass fraction of water from the gravimetric sample preparation was used. The stoichiometry of the mean unknown component $\tilde{U}$ and the estimated composition of all studied mixtures are given in the Supporting Information. 
Table 2: Assignment of chemical groups from GC-COSMO-RS (OL) to ${ }^{13} \mathrm{C}$ NMR chemical shift regions used for the predictions with NEAT in this work. The abbreviated group labels are introduced for clarity. The numbers in parentheses correspond to the group identifiers in the original paper 22 .

\begin{tabular}{l|l|l}
\hline \hline${ }^{13} \mathrm{C}$ NMR chemical shift region & chemical group name & GC-COSMO-RS (OL) label \\
\hline $0-30$ & methyl group & 'CH3' (1) \\
$30-60$ & methylene group & 'CH2' (4) \\
$60-90$ & alcohol group & 'CH2' (7) ${ }^{\mathrm{a}}+$ 'OH(P)' (35) \\
$90-150$ & alkenyl group & 'CH=CH' (58) \\
$150-180$ & carboxyl group & 'COOH' (44) \\
$>180$ & carbonyl group & 'CO' (51) \\
n.a. & water & n.a. \\
\hline \hline
\end{tabular}

${ }^{\text {a }}$ In GC-COSMO-RS (OL), a 'CH2' group bound to $\mathrm{F} / \mathrm{CL} / \mathrm{O} / \mathrm{N}$ is distinguished from a custom 'CH2' group.

Table 3: Assignment of chemical groups from the UNIFAC (DO) table to ${ }^{13} \mathrm{C}$ NMR chemical shift regions used for the predictions with NEAT in this work. The numbers in parentheses are the identifiers for the subgroup and the corresponding main group from the original papers 1 3 .

\begin{tabular}{|c|c|c|}
\hline${ }^{13} \mathrm{C}$ NMR chemical shift region & chemical group name & UNIFAC (DO) label \\
\hline $0-30$ & methyl group & 'CH3' $(1,1)$ \\
\hline $30-60$ & methylene group & 'CH2' $(2,1)$ \\
\hline $60-90$ & alcohol group & 'CH2' $(2,1)+{ }^{\prime} \mathrm{OH}(\mathrm{P}) '(14,5)$ \\
\hline $90-150$ & alkenyl group & 'CH=CH' $(6,2)$ \\
\hline $150-180$ & carboxyl group & 'COOH' $(42,20)$ \\
\hline$>180$ & carbonyl group & 'CH2CO' $(19,9)$ \\
\hline n.a. & water & 'H2O' $(16,7)$ \\
\hline
\end{tabular}




\subsubsection{Calculation of activity coefficients}

After estimating the groups of the mean unknown component $\tilde{U}$, each poorly specified mixture is considered as pseudo-ternary mixture of the components target component $\mathrm{T}+$ mean unknown component $\tilde{\mathrm{U}}+$ water $\mathrm{W}$. In the following, the procedure for the calculation of the activity coefficient $\gamma_{\mathrm{T}}$ of the target component $\mathrm{T}$ in the poorly specified mixture based on a combination of NMR spectroscopy and GC-COSMO-RS (OL) is described. The procedure for the application of NEAT based on UNIFAC (DO) is the same as in our previous work 18 and therefore not discussed here. In COSMO-RS (OL), $\sigma$ profiles and cavity surface areas for the hydrogen bonding (hb) part, and the non-hydrogen bonding (nhb) part are considered [21, 22, which are denoted here as $p^{\mathrm{hb}}(\sigma), p^{\mathrm{nhb}}(\sigma)$ and $A^{\mathrm{hb}}, A^{\mathrm{nhb}}$ respectively. Furthermore, in COSMORS (OL), activity coefficients are modeled as the sum of two contributions, a residual and a combinatorial part. The residual part of the activity coefficient of a component is calculated from its cavity surface areas $A^{\mathrm{hb}}$ and $A^{\mathrm{nhb}}$ and the sigma-profiles $p^{\mathrm{hb}}(\sigma)$ and $p^{\mathrm{nhb}}(\sigma)$ of all pure components that make up the mixture. The combinatorial part is calculated from the cavity volumes and the cavity surface areas of the pure components. For the target component $\mathrm{T}$ and water $\mathrm{W}, \sigma$-profiles, cavity surface areas $A$, and cavity volumes $V$ from quantum-chemical calculations were used, which were taken from the Dortmund Data Bank, 2018, www.ddbst.com (DDB) 23]. For the mean unknown component $\tilde{U}$, the corresponding numbers were obtained from a summation of the contributions of the estimated groups of $\tilde{U}$ using the available group parameters of GC-COSMO-RS (OL) reported in the DDB. Corrections that were introduced in GC-COSMO-RS (OL) to consider intramolecular interactions, such as intramolecular hydrogen bonds or steric effects, were neglected in the present version of NEAT. For the results based on the fully specified mixture, which were only used for comparison here, the $\sigma$-profiles, cavity surface areas $A$ and cavity volumes $V$ of the unknown components $\mathrm{U}$ were calculated as described above, whereas for the target component $\mathrm{T}$ and water $\mathrm{W}$, the numbers were taken from 
the DDB, if not stated otherwise. The calculations of all activity coefficients were carried out using the COSMO-RS (OL) tool supplied with the installation of the DDB. Computational details are given in the Supporting Information.

\section{Results and Discussion}

For simplicity, only activity coefficients at $T=298 \mathrm{~K}$ and $p=1$ bar are considered here. The pressure is not specified in the following as it has only a weak influence on the activity coefficient and is not considered in the studied $G^{\mathrm{E}}$ models. In the following, the term 'system' refers to a set of components without specifying the composition whereas the term 'mixture' is used if additionally the composition is specified. For each system, several mixtures were studied. Table 4 gives an overview of the systems that were investigated in the present work. In all following figures, lines denote the results that were obtained using the full knowledge on the speciation of the mixture, whereas the symbols denote the predictions with NEAT, for which only the information on the target component $\mathrm{T}$ was used, if not stated otherwise. The predictions with NEAT are based on GC-COSMO-RS (OL), if not stated otherwise. In Figure 1, results for the activity coefficient of two target components $(\mathrm{T}=$ acetone or $\mathrm{T}=$ acetic acid $)$ in ternary aqueous mixtures of system I and II are shown. In all cases, 2propanol is considered to be the unknown component $\mathrm{U}$. The results for the fully specified mixtures show a strong influence of $\mathrm{U}$ on $\gamma_{\mathrm{T}}$ for $\mathrm{T}=$ acetone and a rather weak influence for $\mathrm{T}=$ acetic acid. For all mixtures, excellent agreement of the prediction with NEAT with the results for the fully specified mixtures is observed. In Figure S.4 in the Supporting Information, it is shown that the assumed molar mass $M_{\tilde{\mathrm{U}}}$ of the mean unknown component $\tilde{\mathrm{U}}$, which was set to $M_{\tilde{\mathrm{U}}}=150 \mathrm{~g} \mathrm{~mol}^{-1}$ for the results in Figure 1, has only a minor influence on the predictions with NEAT, if no unreasonably small values of $M_{\tilde{\mathrm{U}}}$ are chosen. This was found for all studied mixtures as well as in our previous works [17, 18]. Therefore, only predictions based on $M_{\tilde{\mathrm{U}}}=150 \mathrm{~g} \mathrm{~mol}^{-1}$ are shown in the following. In Figure S.18 in the Supporting Information, we demonstrate that 
Table 4: Overview of the studied systems. Besides the target component $\mathrm{T}$ and the unknown components $\mathrm{U}$, water $\mathrm{W}$ is always present in the mixtures. In all mixtures of a given system, the molar ratio of the target component to water $n_{\mathrm{T}} / n_{\mathrm{W}}$ is constant. For all five-component systems, the mass ratio of the three unknown components is 1:1:1 in all mixtures.

\begin{tabular}{lllll}
\hline System & Target component T & Unknown component(s) $\mathrm{U}$ & $n_{\mathrm{T}} / n_{\mathrm{W}}$ & NMR data \\
\hline I & acetone & 2-propanol & 0.040 & This work \\
\hline II & acetic acid & 2-propanol & 0.050 & This work \\
\hline III & ethanol & acetic acid & 0.043 & Ref. [17] \\
\hline IV & ethanol & methyl acetate & 0.044 & Ref. [17] \\
\hline V & ethanol & 2-butanone & 0.044 & Ref. [17] \\
\hline VI & ethanol & cyclohexanone & 0.044 & This work \\
\hline & & cyclohexanone & & \\
VII & 1,4-butanediol & acetonitrile & 0.022 & Ref. [17] \\
& & methyl acetate & & \\
\hline VIII & acetone & D-xylose & & \\
& & acetic acid & & \\
\hline \hline
\end{tabular}


NEAT is not restricted to a prediction at $T=298 \mathrm{~K}$, i.e. the temperature at which the NMR analysis is carried out, but also gives good results for other temperatures. This is not surprising, since GC-COSMO-RS (OL), which is the basis of the NEAT version here, takes the temperature dependence of activity coefficients into account.

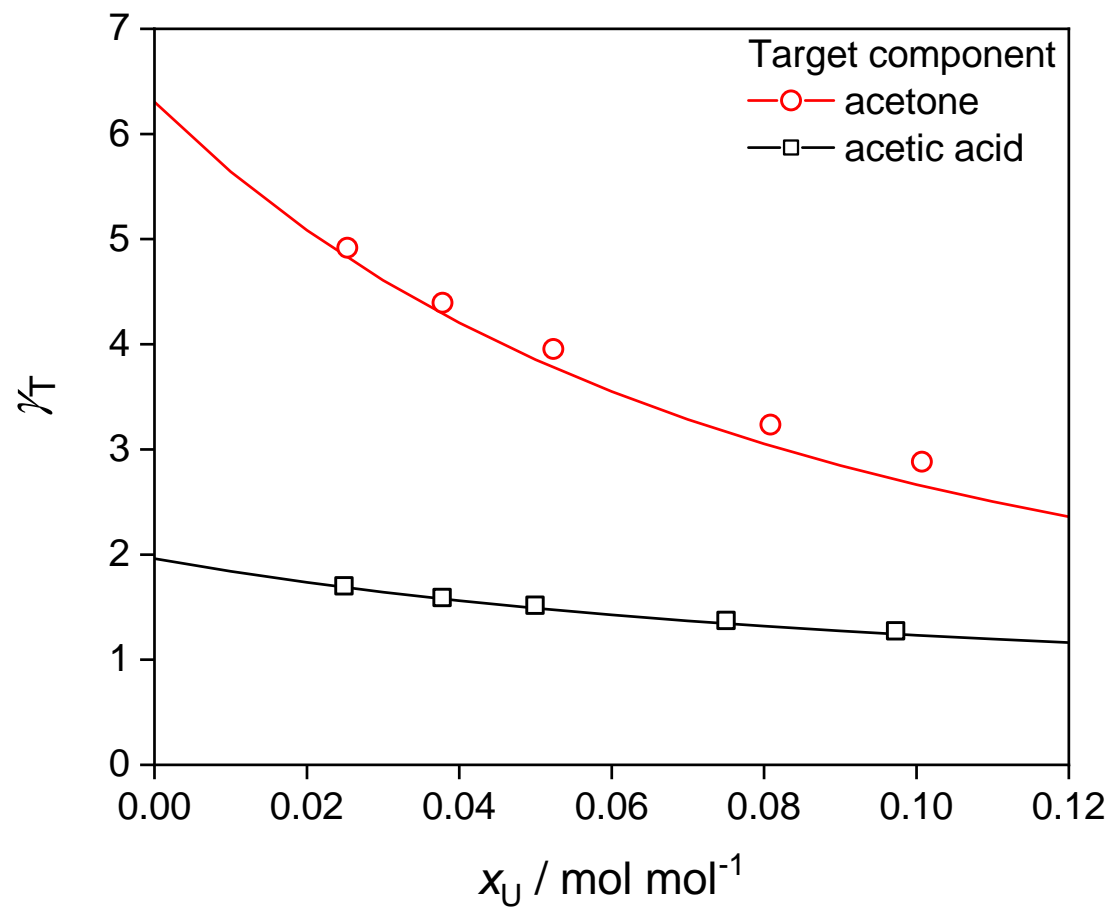

Figure 1: Activity coefficient $\gamma_{\mathrm{T}}$ of target components $(\mathrm{T}=$ acetone or $\mathrm{T}=$ acetic acid) in ternary mixtures of system I and II, cf. Table 4 at 298 K. Lines: results from GC-COSMORS (OL) for the fully specified mixtures. Symbols: predictions with NEAT. No information on the unknown component $\mathrm{U}$ was used in NEAT.

Figure 2 shows the $\sigma$-profile of the mean unknown component $\tilde{U}$ in a mixture of system I $\left(x_{\mathrm{U}}=0.025 \mathrm{~mol} \mathrm{~mol}^{-1}\right)$ as predicted by NEAT based on the NMR analysis of the mixture. For comparison, also the $\sigma$-profile of $\mathrm{U}=2$-propanol is shown. The agreement of the $\sigma$-profiles is very good, which explains the excellent prediction of $\gamma_{\mathrm{T}}$ in Figure 1 The respective plot for a mixture of system II is given in Figure S.5 in the Supporting Information and shows a 
similar agreement.

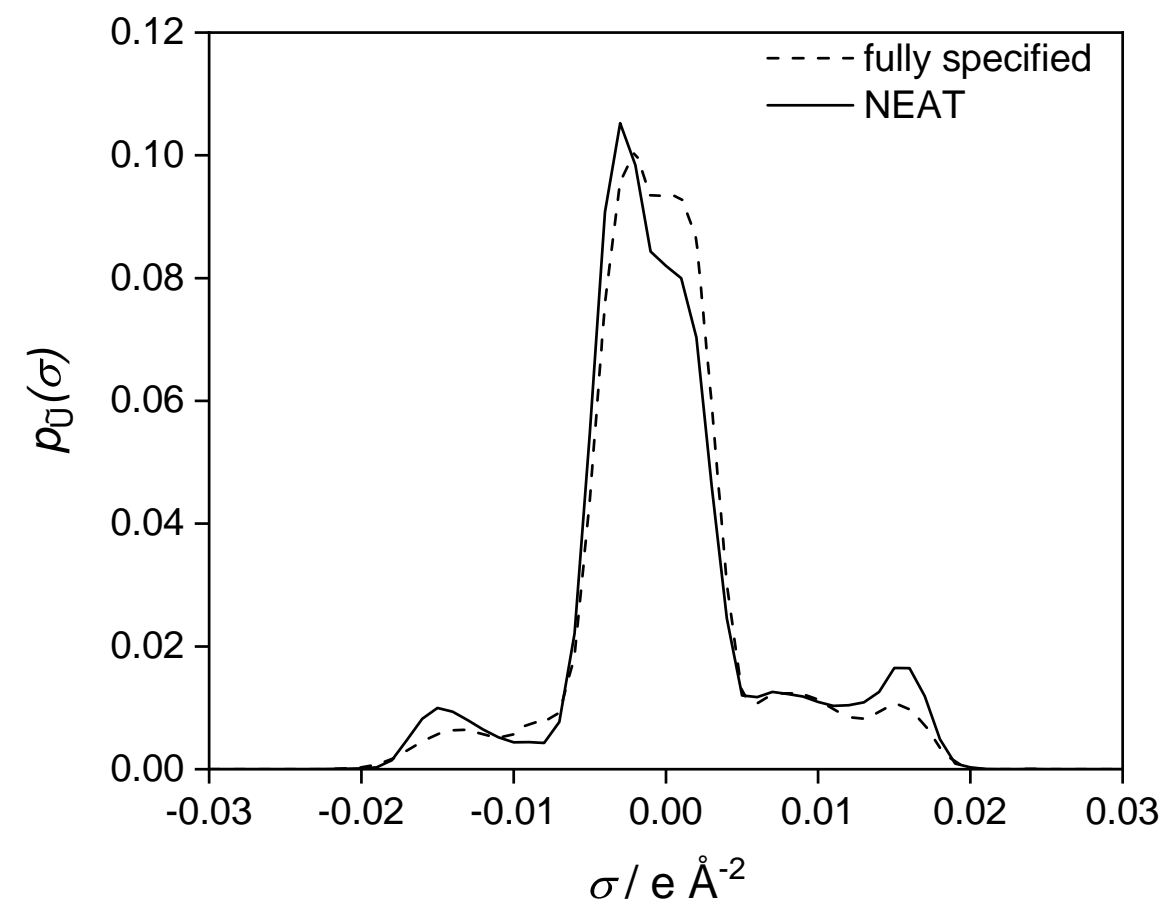

Figure 2: $\sigma$-profile of the mean unknown component $\tilde{U}$ in a mixture of system I as predicted with NEAT and the $\sigma$-profile of $\mathrm{U}=2$-propanol. $p_{\tilde{\mathrm{U}}}(\sigma)$ is the cavity surface area-weighted sum of $p_{\tilde{\mathrm{U}}}^{\mathrm{nhb}}(\sigma)$ and $p_{\tilde{\mathrm{U}}}^{\mathrm{hb}}(\sigma)$, cf. Supporting Information. Dashed line: result from GCCOSMO-RS (OL) for U in the fully specified mixture. Solid line: prediction with NEAT. The composition of the mixture is $x_{\mathrm{T}}=0.037 \mathrm{~mol} \mathrm{~mol}^{-1}, x_{\mathrm{U}}=0.025 \mathrm{~mol} \mathrm{~mol}^{-1}$.

In Figure 3 , the influence of different unknown components $\mathrm{U}$ on the activity coefficient $\gamma_{\mathrm{T}}$ of the target component $\mathrm{T}=$ ethanol is studied. The unknown components are $\mathrm{U}=$ acetic acid, $\mathrm{U}=$ methyl acetate, $\mathrm{U}=2$-butanone, and $\mathrm{U}=$ cyclohexanone (systems III - VI, cf. Table 4). The studied compositions were limited by the solubilities in the respective systems. The results show that acetic acid has a weak influence on $\gamma_{\mathrm{T}}$, whereas cyclohexanone has a strong influence. Methyl acetate and 2-butanone show a medium influence. For all unknown components $\mathrm{U}$, the agreement of the predictions with NEAT with the results for the fully specified mixture is very good. In particular, the differences 
in the strength of the influence of the different unknown components $\mathrm{U}$ on $\gamma_{\mathrm{T}}$ are predicted well with NEAT.

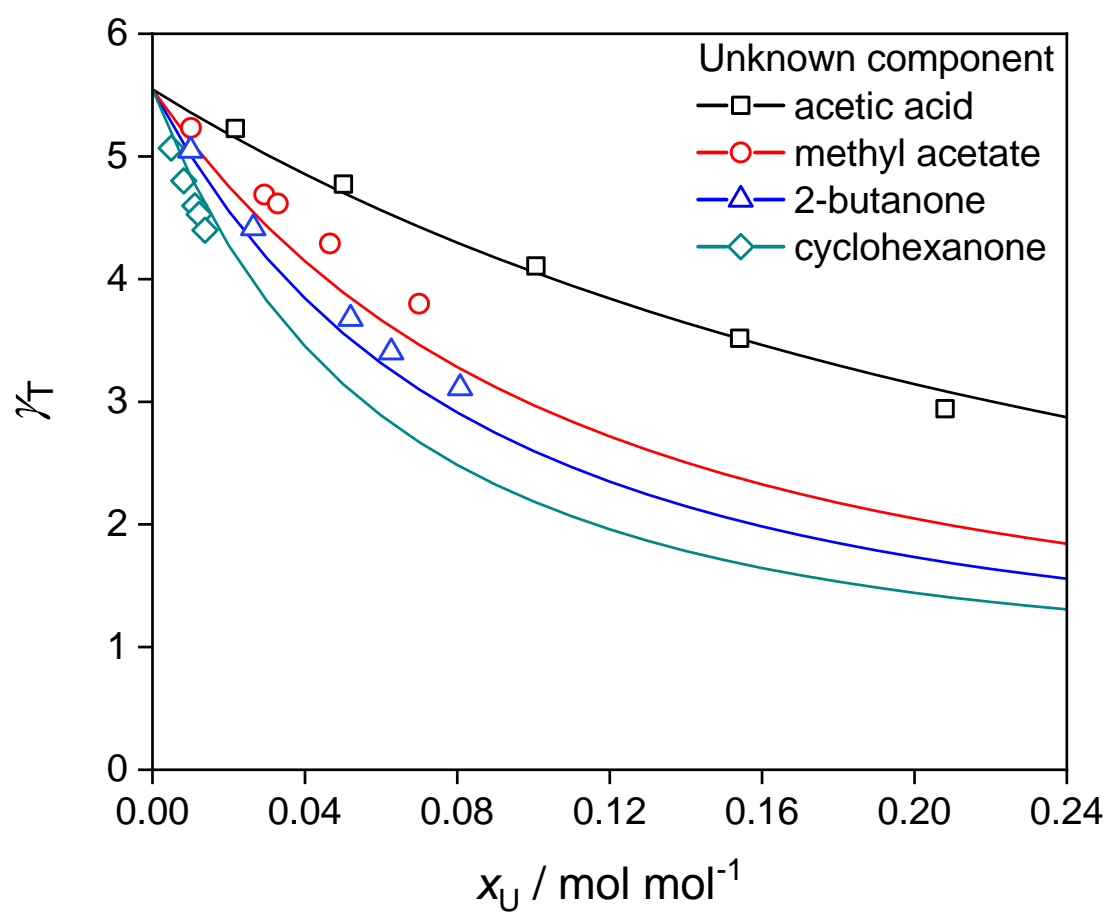

Figure 3: Activity coefficient $\gamma_{\mathrm{T}}$ of target component $\mathrm{T}=$ ethanol in ternary mixtures of systems III - VI, cf. Table 4 at 298 K. Lines: results from GC-COSMO-RS (OL) for the fully specified mixtures. Symbols: predictions with NEAT. No information on the unknown component $\mathrm{U}$ was used in NEAT.

Figure 4 shows the $\sigma$-profile of the mean unknown component $\tilde{U}$ in a mixture

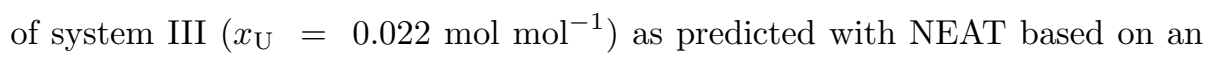
NMR analysis of the mixture. For comparison, also the $\sigma$-profile of $\mathrm{U}=$ acetic acid is shown. The agreement of the $\sigma$-profiles is excellent, which is in line with the excellent prediction of $\gamma_{\mathrm{T}}$ in Figure 3. The respective plots for systems IV 245 - VI are given in the Figures S.6-S.8 in the Supporting Information and show a similar agreement. 


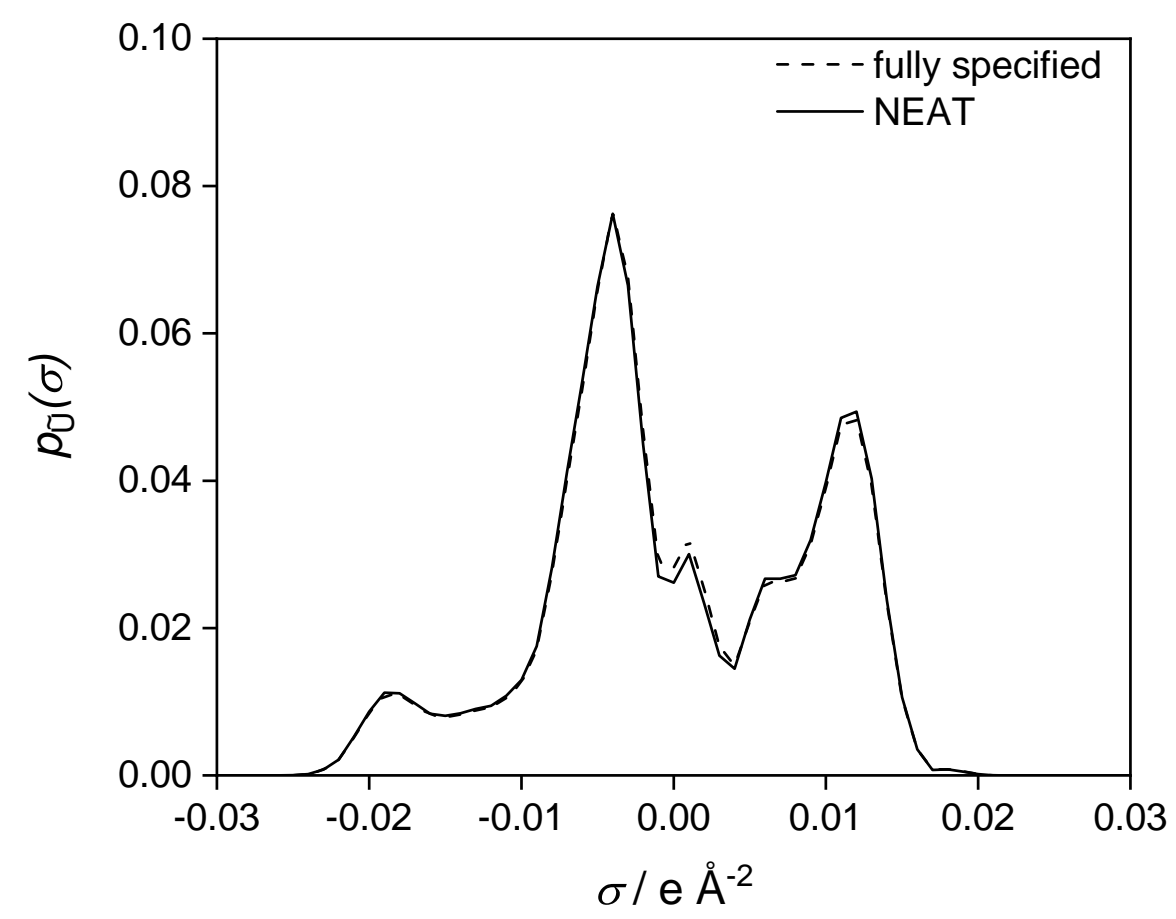

Figure 4: $\sigma$-profile of the mean unknown component $\tilde{U}$ in a mixture of system III as predicted with NEAT and the $\sigma$-profile of $\mathrm{U}=$ acetic acid. $p_{\tilde{\mathrm{U}}}(\sigma)$ is the cavity surface area-weighted sum of $p_{\tilde{\mathrm{U}}}^{\mathrm{nhb}}(\sigma)$ and $p_{\tilde{\mathrm{U}}}^{\mathrm{hb}}(\sigma)$, cf. Supporting Information. Dashed line: result from GCCOSMO-RS (OL) for U in the fully specified mixture. Solid line: prediction with NEAT. The composition of the mixture is $x_{\mathrm{T}}=0.041 \mathrm{~mol} \mathrm{~mol}^{-1}, x_{\mathrm{U}}=0.022 \mathrm{~mol} \mathrm{~mol}^{-1}$.

In Figure 5, results for the activity coefficient of two target components ( $\mathrm{T}=1$,4-butanediol or $\mathrm{T}=$ acetone) for aqueous five-component mixtures containing the unknown components $\mathrm{U}=$ cyclohexanone, acetonitrile, methyl acetate or $\mathrm{U}=\mathrm{D}$-xylose, acetic acid, methyl acetate are shown. For D-xylose, GCCOSMO-RS (OL) cannot be used in its current version since the required parameters are not available. Therefore, for obtaining the results for system VIII, quantum-chemical obtained $\sigma$-profiles, cavity surface areas $A$, and the cavity volume $V$ from the DDB were used for D-xylose for the fully specified mixture.

255 In Figure 5, results for two versions of NEAT are shown, one that is based on GC-COSMO-RS (OL) and one that is based on UNIFAC (DO). Both predic- 
tions with NEAT are compared to the results for the fully specified mixture that were obtained with the respective thermodynamic model. For system VIII, the results from GC-COSMO-RS (OL) and UNIFAC (DO) are similar, whereas there is a large deviation for system VII. However, it is not the intention of the present work to evaluate and compare the suitability of GC-COSMO-RS (OL) and UNIFAC (DO) for the studied mixtures. For both models, the agreement of all predictions with NEAT with the results for the fully specified mixture is excellent. The results demonstrate the generic nature of the idea of NEAT that allows to select the most suitable thermodynamic model for the studied system.

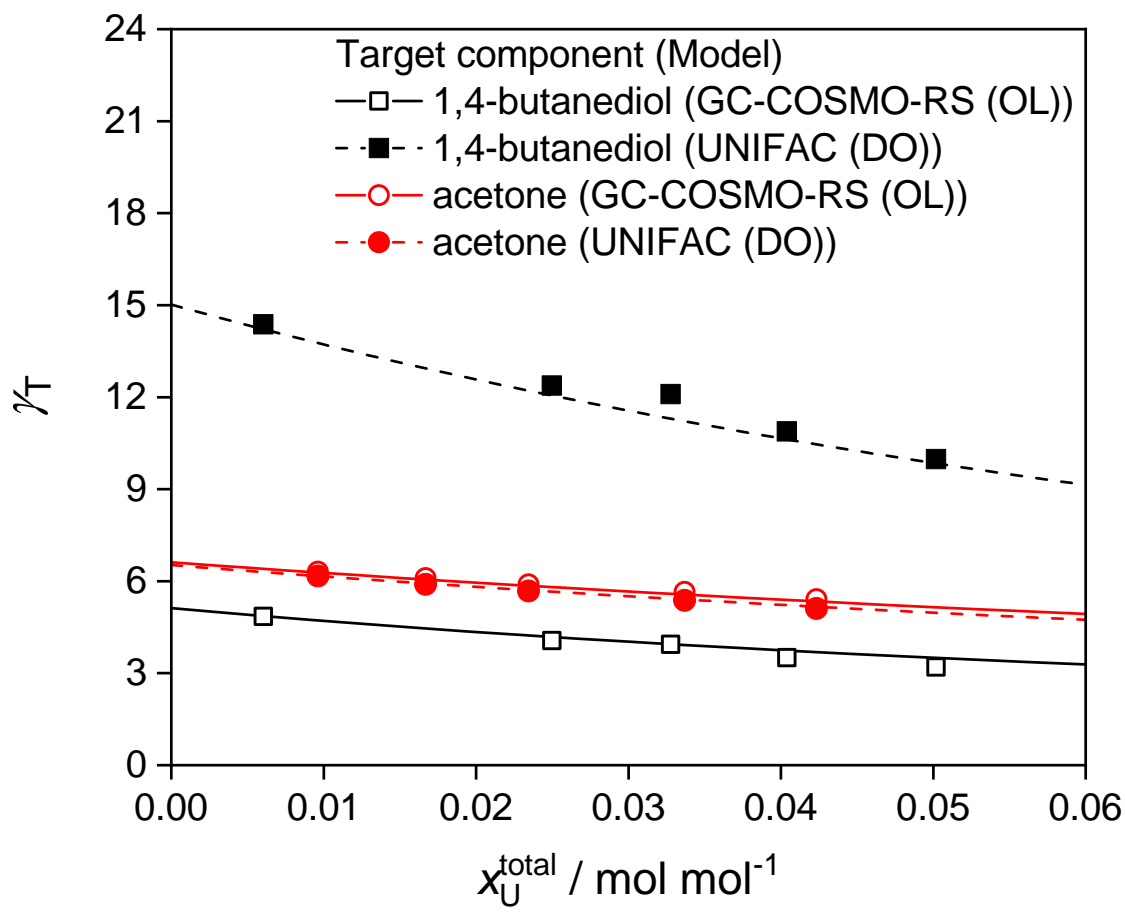

Figure 5: Activity coefficient $\gamma_{\mathrm{T}}$ of target components $(\mathrm{T}=1,4$-butanediol or $\mathrm{T}=$ acetone $)$ in five-component mixtures of system VII and VIII, cf. Table 4 at $298 \mathrm{~K}$. Lines: results for fully specified mixtures. Symbols: predictions with NEAT. No information on the unknown components $\mathrm{U}$ was used in NEAT.

Figure 6 shows the $\sigma$-profiles of the mean unknown component $\tilde{U}$ calcu- 
lated with information from NEAT and for the fully specified mixture in a five-component aqueous mixture of system VII $\left(x_{\mathrm{U}}^{\text {total }}=0.006 \mathrm{~mol} \mathrm{~mol}^{-1}\right)$.

Compared to the results in Figures 2 and 4 , the deviations are larger, which can be attributed to the more complex nature of the mixture. The respective plot for system VIII is given in Figure S.9 in the Supporting Information and shows a similar agreement.

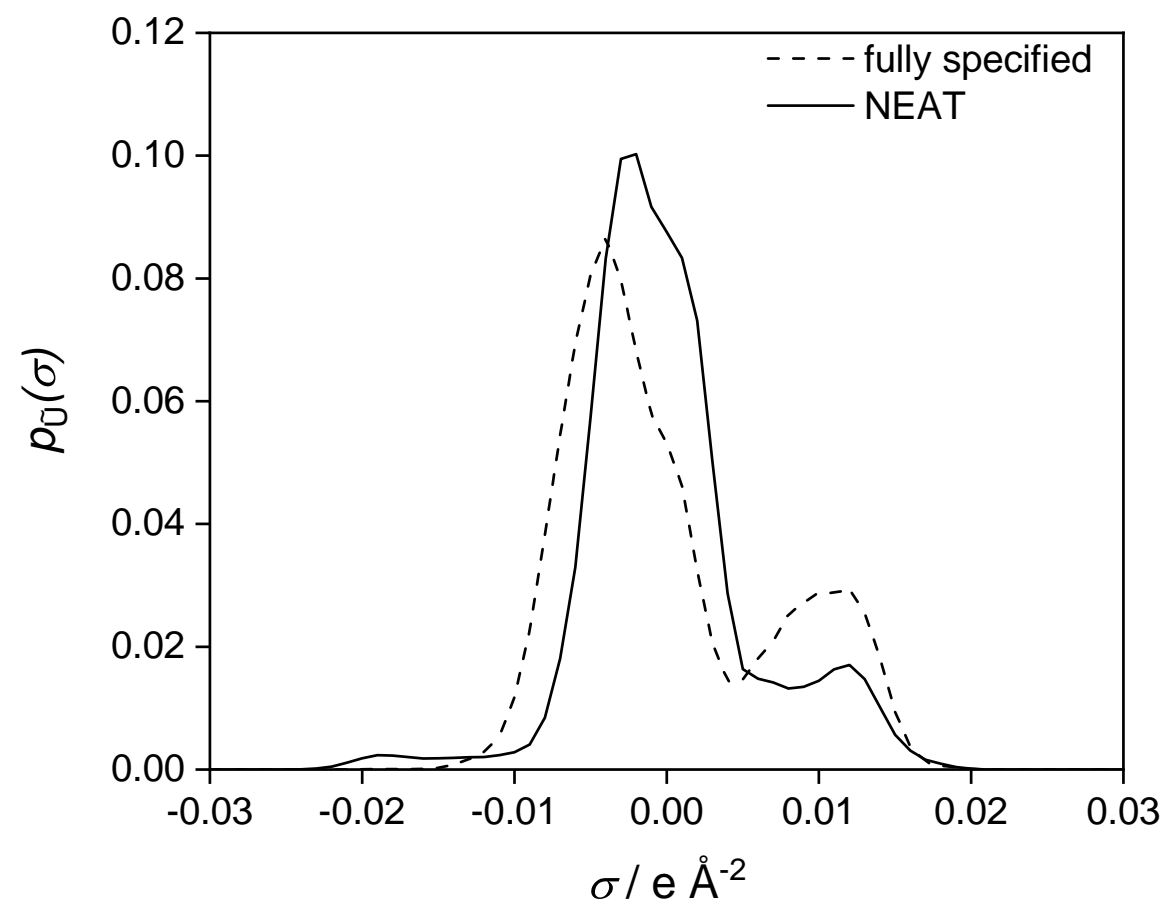

Figure 6: $\sigma$-profile of the mean unknown component $\tilde{U}$ in a mixture of system VII as predicted with NEAT and the mixed $\sigma$-profile of the unknown components $\mathrm{U}_{1}=$ cyclohexanone, $\mathrm{U}_{2}=$ acetonitrile, $\mathrm{U}_{3}=$ methyl acetate. $p_{\tilde{\mathrm{U}}}(\sigma)$ is the cavity surface area-weighted sum of $p_{\tilde{\mathrm{U}}}^{\mathrm{nhb}}(\sigma)$ and $p_{\tilde{\mathrm{U}}}^{\mathrm{hb}}(\sigma)$, cf. Supporting Information. Dashed line: results from GC-COSMORS (OL) for the mixture of all $\mathrm{U}_{i}$ in the fully specified mixture. Solid line: prediction with NEAT. The composition of the mixture is $x_{\mathrm{T}}=0.021 \mathrm{~mol} \mathrm{~mol}^{-1}, x_{\mathrm{U}}^{\text {total }}=0.006 \mathrm{~mol} \mathrm{~mol}^{-1}$. 


\section{Conclusions}

275 and challenging in process design. In a recent work, we have introduced the method NEAT, that enables the prediction of the activity coefficients of target components in poorly specified mixtures based on a combination of NMR spectroscopy and thermodynamic group contribution methods. In all previous 


\section{Declaration of Competing Interest}

The authors declare that they have no known competing financial interests or personal relationships that could have appeared to influence the work reported 305 in this paper.

\section{Appendix A. Supplementary data}

The Supporting Information to this article can be found online at [Link].

\section{Nomenclature}

Symbols

A cavity surface area

$p(\sigma) \quad \sigma$-profile

$p \quad$ pressure

$\mathrm{T} \quad$ target component

$T$ temperature

U unknown component

$\tilde{U}$ mean unknown component

$V \quad$ cavity volume

W water

$x \quad$ mole fraction

Greek letters

$\gamma$ activity coefficient

$\sigma$ screening charge density

Sub- and superscripts 


$$
\begin{array}{ll}
\text { hb } & \text { hydrogen bonding } \\
\text { nhb } & \text { nonhydrogen bonding } \\
i & \text { component } \\
\text { total } & \text { total composition }
\end{array}
$$

\section{Abbreviations}

$\begin{array}{ll}\text { COSMO } & \text { Conductor like Screening Model } \\ \text { COSMO-RS } & \text { Conductor like Screening Model for Real Solvents } \\ \text { NEAT } & \text { NMR Spectroscopy for the Estimation of Activity Coefficients of } \\ & \text { Target Components in Poorly Specified Mixtures } \\ \text { NMR } & \text { Nuclear Magnetic Resonance } \\ \text { TMSP-d4 } & \text { Sodium 3-(trimethylsilyl)tetradeuteriopropionate } \\ \text { UNIFAC } & \text { Universal Quasichemical Functional Group Activity Coefficients } \\ \text { UNIQUAC } & \text { Universal Quasichemical }\end{array}$

\section{References}

[1] A. Fredenslund, J. Gmehling, P. Rasmussen, Vapor-Liquid Equilibria Using UNIFAC, a Group-Contribution Method, Elsevier: Amsterdam, The Netherlands, 1977.

[2] U. Weidlich, J. Gmehling, A modified UNIFAC Model. 1. Prediction of VLE, $h^{\mathrm{E}}$, and $\gamma^{\infty}$., Ind. Eng. Chem. Res. 26 (1987) 1372-1381. https://doi.org/10.1021/ie00067a018.

[3] D. Constantinescu, J. Gmehling, Further Development of Modified UNIFAC (Dortmund): Revision and Extension 6, J. Chem. Eng. Data 61 (2016) 2738-2748. https://doi.org/10.1021/acs.jced.6b00136.

[4] A. Klamt, Conductor-like Screening Model for Real Solvents: A New Approach to the Quantitative Calculation of Solvation Phenomena, J. Phys. Chem. 99 (1995) 2224-2235. https://doi.org/10.1021/j100007a062. 
[5] A. Klamt, V. Jonas, T. Bürger, J. C. W. Lohrenz, Refinement and Parametrization of COSMO-RS, J. Phys. Chem. A 102 (1998) 5074-5085. https://doi.org/10.1021/jp980017s.

[6] D. S. Abrams, J. M. Prausnitz, Statistical Thermodynamics of Liquid Mixtures: A New Expression for the Excess Gibbs Energy of Partly or Completely Miscible Systems, AIChE J. 21 (1975) 116-128. https://doi.org/10.1002/aic.690210115.

[7] A. Klamt, G. Schüürmann, COSMO: A New Approach to Dielectric Screening in Solvents with Explicit Expressions for the Screening Energy and its Gradient, J. Chem. Soc., Perkin Trans. 2 (1993) 799-805. https://doi.org/10.1039/P29930000799.

[8] G. L. Alexander, A. L. Creagh, J. M. Prausnitz, Phase Equilibriums for High-Boiling Fossil Fuel Distillates. 1. Characterization, I\&EC Fundam. 24 (1985) 301-310. https://doi.org/10.1021/i100019a005.

[9] V. Ruzicka Jr., A. Fredenslund, P. Rasmussen, Representation of Petroleum Fractions by Group Contribution, Ind. Eng. Chem. Process Des. Dev. 22 (1983) 49-53. https://doi.org/10.1021/i200020a009.

[10] G. L. Alexander, B. J. Schwarz, J. M. Prausnitz, Phase Equilibriums for High-Boiling Fossil Fuel Distillates. 2. Correlation of Equation-of-State Constants with Characterization Data for Phase Equilibrium Calculations, I\&EC Fundam. 24 (1985) 311-315. https://doi.org/10.1021/i100019a006.

[11] H. Hartounian, D. T. Allen, Group Contribution Methods for Coal-derived Liquids: Hydrogen Solubilities using a UNIFAC Approach, Fuel 67 (1988) 1609-1614. https://doi.org/10.1016/0016-2361(88)90203-7.

[12] A. G. Abdul Jameel, A. M. Elbaz, A.-H. Emwas, W. L. Roberts, S. M. Sarathy, Calculation of Average Molecular Parameters, Functional Groups, and a Surrogate Molecule for Heavy Fuel Oils Using ${ }^{1} \mathrm{H}$ and ${ }^{13} \mathrm{C} \mathrm{Nu}-$ 
clear Magnetic Resonance Spectroscopy, Energy Fuels 30 (2016) 3894-3905. https://doi.org/10.1021/acs.energyfuels.6b00303.

[13] E. I. Darwish, T. A. Al-Sahhaf, M. A. Fahim, Prediction of the Surface Tension of Petroleum Cuts using a Modified UNIFAC Group Contribution Method, Fluid Phase Equilib. 105 (1995) 229-239. https://doi.org/10.1016/0378-3812(94)02619-C.

[14] B. Carreón-Calderón, V. Uribe-Vargas, E. Ramírez-Jaramillo, M. Ramírezde Santiago, Thermodynamic Characterization of Undefined Petroleum Fractions using Group Contribution Methods, Ind. Eng. Chem. Res. 51 (2012) 14188-14198. https://doi.org/10.1021/ie3016076.

[15] B. Carreón-Calderón, V. Uribe-Vargas, M. Ramírez-de Santiago, E. Ramírez-Jaramillo, Thermodynamic Characterization of Heavy Petroleum Fluids using Group Contribution Methods, Ind. Eng. Chem. Res. 53 (2014) 5598-5607. https://doi.org/10.1021/ie403967z.

[16] T. Thuy, D. T. Allen, Property Estimation using Structural Characterizations: Heat Capacities of Coal Liquids, Fuel 64 (1985) 1754-1759. https://doi.org/10.1016/0016-2361(85)90404-1.

[17] F. Jirasek, J. Burger, H. Hasse, Method for Estimating Activity Coefficients of Target Components in Poorly Specified Mixtures, Ind. Eng. Chem. Res. 57 (2018) 7310-7313. https://doi.org/10.1021/acs.iecr.8b00917.

[18] F. Jirasek, J. Burger, H. Hasse, NEAT-NMR Spectroscopy for the Estimation of Activity Coefficients of Target Components in Poorly 375 Specified Mixtures, Ind. Eng. Chem. Res. 58 (2019) 9155-9165. https://doi.org/10.1021/acs.iecr.9b01269.

[19] F. Jirasek, J. Burger, H. Hasse, Application of NEAT for Determining the Composition Dependence of Activity Coefficients in Poorly Specified Mixtures, Chem. Eng. Sci. 208 (2019) 115161. https://doi.org/10.1016/j.ces.2019.115161. 
[20] F. Jirasek, J. Burger, H. Hasse, Application of NEAT for the Simulation of Liquid-liquid Extraction Processes with Poorly Specified Feeds, AIChE J. (2019) e16826. https://doi.org/10.1002/aic.16826.

[21] H. Grensemann, J. Gmehling, Performance of a Conductor-Like Screening Model for Real Solvents Model in Comparison to Classical Group Contribution Methods, Ind. Eng. Chem. Res. 44 (2005) 1610-1624. https://doi.org/10.1021/ie049139z.

[22] T. Mu, J. Rarey, J. Gmehling, Group Contribution Prediction of Surface Charge Density Profiles for COSMO-RS(Ol), AIChE J. 53 (2007) 32313240. https://doi.org/10.1002/aic.11338.

[23] Dortmund data bank 2018, www.ddbst.com. 